九州大学学術情報リポジトリ

Kyushu University Institutional Repository

\title{
Cue integration as a common mechanism for action and outcome bindings
}

Yamamoto, Kentaro

Faculty of Human-Environment Studies, Kyushu University : Lecturer

http://hdl. handle. net/2324/4751313

出版情報: Cognition : international journal of cognitive psychology. 205 (104423), 2020-08-22. Elsevier B.V.

バージョン:

権利関係：CC BY-NC-ND 
5 aFaculty of Human-Environment Studies, Kyushu University, Fukuoka, Japan

6 bFaculty of Science and Engineering, Waseda University, Tokyo, Japan

9 Running head: CUE INTEGRATION AND INTENTIONAL BINDING

$11{ }^{*}$ Correspondence:

12 Dr. Kentaro Yamamoto

13 Faculty of Human-Environment Studies, Kyushu University,

14744 Motoka, Nishi-ku, Fukuoka-shi, Fukuoka 819-0395, Japan

15 E-mail: yamamoto.kntr@hes.kyushu-u.ac.jp

16 Tel: +81-92-802-5173

17

18 Note: This is an accepted version of a paper published in Cognition The final publication is available at https://doi.org/10.1016/j.cognition.2020.104423 


\section{Abstract}

25 When a voluntary action is followed by a sensory outcome, their timings are perceived 26 to shift toward each other compared to when they were generated independently. Recent

27 studies have tried to explain this temporal binding effect based on the cue integration

28 theory, in which the timing of action and outcome are estimated as a precision-weighted 29 average of their individual estimates, although distinct results were obtained between

30 the binding of action and outcome. This study demonstrates that cue integration

31 underlies both action and outcome bindings, using visual changes as action outcomes.

32 Participants viewed a moving clock presented on a screen to report the onset time of

33 their action or the feature changes of visual objects that were relevant or irrelevant to

34 the clock movement. The results revealed that the precision of outcome timing

35 judgment was different based on the object that underwent a feature change. Moreover,

36 consistent with the theory's prediction, the perceptual shifts of action and outcome

37 timings were larger and smaller, respectively, when the precision of outcome timing

38 judgments was higher. These results suggest that cue integration serves as a common 39 mechanism in action and outcome bindings.

40

41 Keywords: subjective time, intentional binding, action, visual outcome, cue integration 
42

43

44

45

\section{Introduction}

The temporal relationship between action and its sensory outcome is important in understanding their causal linkage and in experiencing a sense of agency. If a sensory event occurs shortly after an action was taken, it is natural to assume that the action triggered the event. It was recently suggested that the temporal judgment of action and its outcome is similarly dependent on the causal relationship. Previous studies have demonstrated that, when a sensory outcome (e.g., auditory tone) was triggered by a voluntary action, the timing of the action appeared to be later, while that of the outcome appeared to be earlier, relative to when they were generated independently (e.g., Haggard \& Clark, 2003; Haggard, Clark, \& Kalogeras, 2002). Interestingly, this temporal attraction between action and outcome occurred when the action was intentional, but the perceptual changes were reversed when the action was produced involuntarily via magnetic brain stimulation (Haggard et al., 2002). This phenomenon, known as intentional binding, suggests that the perception of event timing is reconstructed through the experience of agency.

Previous studies have mainly focused on what factors influence intentional binding. For example, Engbert and Wohlschläger (2007) manipulated the ratio of the occurrence of an effect (i.e., a tone) and found that the binding effect was stronger when the tone occurred with a higher probability, relative to that observed when the tone occurred with a lower probability. This indicated that the predictability of action outcome was important for temporal binding. Temporal contiguity and predictability also appeared to influence temporal binding (Cravo, Claessens, \& Baldo, 2011; Haggard et al., 2002). The aforementioned studies showed that the binding effect became stronger when the interval between action and outcome was short (e.g., $250 \mathrm{~ms}$ ) and 
66

67

fixed. Moreover, Desantis, Roussel, and Waszak (2011) showed that the binding effect was stronger when participants believed that their actions triggered outcomes, relative to that observed when they believed that another person had triggered the outcome. This result suggested that causal beliefs about agency dominated intentional binding. Overall, the findings of these studies indicate that intentional binding depends largely on the causality of action effects. However, the mechanism underlying this phenomenon remains unclear.

Cue integration has recently been suggested to underlie intentional binding (Kawabe, Roseboom, \& Nishida, 2013; Kirsch, Kunde, Herbort, 2019; Lush et al., in press; Wolpe, Haggard, Siebner, \& Rowe, 2013). This is based on the idea that the perceptual system optimally combines different sensory information to produce more reliable estimates, which is well-documented in multisensory perception literature (e.g., Alais \& Burr, 2004; Ernst \& Bülthoff, 2004). According to this view, the timing of each event is estimated as a weighted average of its temporal cues, whereby the weight of each cue is determined by its relative precision. This precision is defined as the inverse of variability of the estimate and is also referred to as sensory "reliability." It is believed that intentional binding occurs when temporal information is further integrated across the events, which are physically interleaved in time (Figure 1a). Importantly, this mechanism predicts that increased precision of one event's timing would result in weaker binding of that event and stronger binding of another. 


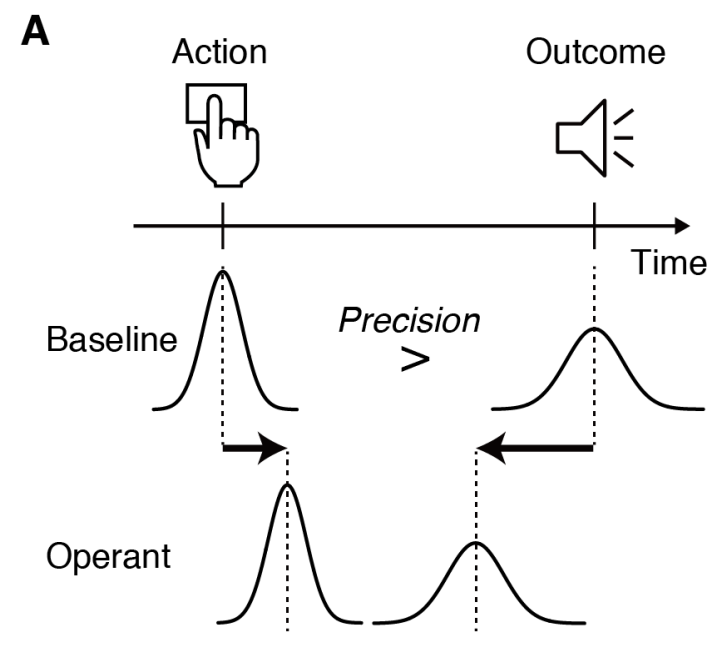

B

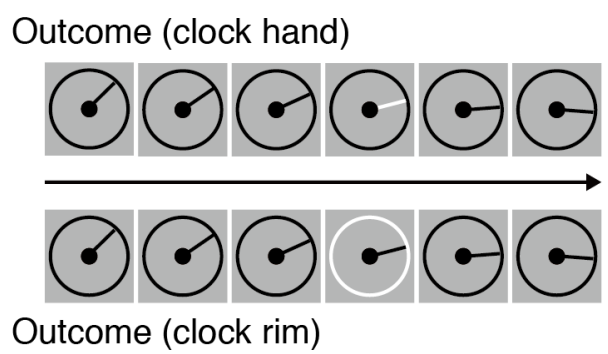

Figure 1. (A) A cue integration account of intentional binding. The temporal cues in each event are integrated to constitute the perceptual estimate. Intentional binding occurs when the cues are further integrated across the events in the operant condition. The relative precision of the estimate is supposed to determine the relative strength of each binding. (B) The two types of visual outcome used in this study. The luminance feature of either clock hand or clock rim changed as action outcome. The precision of the outcome timing judgment was expected to be higher when the change occurred to the moving clock hand.

Several studies have empirically examined the cue integration hypothesis based

87 on this prediction. For example, Wolpe et al. (2013) manipulated the uncertainty of outcome tones by modulating tone intensity to examine its impact on the binding effect.

89 The results showed that, consistent with this prediction, temporal binding of action and outcome became stronger and weaker, respectively, as the precision of outcome timing

91 judgment increased. However, the change in outcome binding was derived from the

92 difference in the perceived timing between baseline conditions, in which an outcome tone was presented without action. Thus, they concluded that action and outcome 
94 binding may be driven by distinct mechanisms. Lush et al. (in press) also examined the

contribution of cue integration, using trait differences in hypnotisability. They showed that greater precision of action timing judgments resulted in reduced action binding, with no difference in outcome binding. These findings indicate that the relative precision of timing judgment was reflected in action binding but not in outcome binding, suggesting the partial contribution of cue integration to intentional binding. However, considering that sensory integration is an automatic process (Helbig \& Ernst, 2008), it is not reasonable to assume that only action binding is driven by actionoutcome cue integration. Indeed, the results of Wolpe et al. (2013) do not contradict the cue integration account of outcome binding when focusing on the binding measures. Although they modulated tone intensity to manipulate the precision of outcome timing judgment, this might have caused the difference in perceived timing between baseline conditions because stimulus intensity can influence response and perceptual latencies (Nissen, 1977). Therefore, it is important to find ways to manipulate the precision of timing judgment without changing stimulus intensity to accurately verify the contribution of cue integration.

In this study, I used visual feature changes as sensory outcomes and manipulated the precision of outcome judgments by varying where the feature changed. In the clock paradigm, which has been used to measure binding, participants watch a moving clock hand to estimate the timing of both an action and its outcome. In this case, sensory signals derived from the events must be combined with the time-varying visual orientation signals of the clock hand to constitute the temporal cues, indicating that temporal uncertainty of the feature binding can influence the precision of timing judgment. Previous studies have shown that dividing attention across features of the 
118

119

120

121

122

123

124

125

126

127

128

129

130

131

132

133

134

135

136

137

138

139

140

same object leads to better performance relative to that observed when dividing

attention across different objects (e.g., Duncan, 1984). Moreover, it has been suggested

that temporal resolution of feature binding is better for spatially superimposed than for spatially separated features (Holcombe \& Cavanagh, 2001). Given these findings, the precision of outcome timing judgment was expected to be higher with visual feature changes in objects that are relevant, rather than irrelevant, to the clock hand. Based on this idea, I tested two visual conditions, in which the rim or moving hand of a clock flashed (Figure 1b), and examined if the manipulated outcome precision would influence action and outcome bindings.

\section{Methods}

\subsection{Participants}

Sixteen paid volunteers (six men and ten women, mean age $\pm S D=20.9 \pm 1.2$ years) participated in the experiment. The sample size was determined using PANGEA (Westfall, 2016) by computing the statistical power of an interaction effect between judged event and flashed object in ANOVA, which was predicted by the cue integration hypothesis. The power analysis revealed that 16 participants were sufficient to detect the interaction with a medium effect size $(d=0.45)$ and a statistical power of 0.8 . All participants had normal or corrected-to-normal vision, were unaware of the experiment's purpose, and provided written informed consent. The experiment was conducted in accordance with the Declaration of Helsinki.

\subsection{Apparatus}

The stimuli were presented on a 22-inch CRT monitor with a resolution of 1,024

$141 \times 768$ pixels and a refresh rate of $100 \mathrm{~Hz}$, controlled by an Apple Macintosh computer. 
142 A chin rest restrained participants' head movements at a viewing distance of $57 \mathrm{~cm}$ from

143 the display. The stimuli were generated using Matlab (The MathWorks, Natick, MA,

144 USA) with the Psychtoolbox extension (Brainard, 1997; Pelli, 1997).

$145 \quad 2.3$ Stimulus and Procedure

146 The experiment was performed in a darkened room. During the task, a clock

147 stimulus consisting of a black hand $(1.7 \mathrm{~cm}$ in length $)$ and a black rim $(3.5 \mathrm{~cm}$ in

148 diameter) was presented with a central fixation marker on a gray background. The clock

149 hand rotated one revolution every $2,560 \mathrm{~ms}$, and its initial position was determined

150 randomly in each trial. As a sensory outcome of action, either the clock frame or the

151 clock hand flashed from black to white for $100 \mathrm{~ms}$. Participants observed the clock and

152 reported onset times for their actions or visual flashes.

153 Participants were exposed to two baseline and two operant conditions in separate

154 blocks. In the baseline-action condition, participants were asked to press a key at a time

155 of their choosing, while avoiding responding to stereotyped or predetermined times. In

156 the baseline-outcome condition, the visual flash occurred 1,500-4,000 ms after trial

157 initiation. In the operant-action and operant-outcome conditions, the visual flash

158 occurred $250 \mathrm{~ms}$ after voluntary action. The clock disappeared 1,500-2,500 $\mathrm{ms}$ after the

159 estimated event, and another clock with a static hand was presented at the same

160 location. The participants' task was to adjust the position of the clock hand, using a

161 keyboard to report the onset of the designated event. The baseline-action condition

162 consisted of 40 trials, and each remaining condition consisted of 80 trials (i.e., 40 trials

163 for each visual outcome). The two types of visual outcome were presented randomly for

164 each block, with no more than two consecutive presentations. The order of the task

165 context (baseline or operant) and judged event (action or outcome) were 
counterbalanced across participants.

167

168

\section{Results}

The difference between judged and actual position of the clock was calculated and converted into a time unit as a judgement error. Trials of which judgment errors exceeded a range of mean $\pm 2.5 S D$ in each condition were excluded from each

172 participant's dataset as outliers (1.6\% of all the trials). The one-sample Kolmogorov-

173 Smirnov tests revealed that the data in each condition did not significantly deviate from

174 a normal distribution ( $p \mathrm{~s}>.22$ ). The normal quantile-quantile plots in Figure 2a also

175 confirmed the assumption of normality, ensuring the validity of the variability

176 comparison. Table 1 shows the judgment errors and variabilities (i.e., standard

177 deviations ${ }^{1}$ of judgement error across trials) averaged across participants. First, the

178 variability of judgements in the two baseline-outcome conditions were compared. A

179 two-tailed paired $t$-test revealed that there was a significant difference, $t(15)=3.62, p$

$180=.003$, Cohen's $d z=0.90$, indicating that variability when the clock hand flashed was

Table 1. Mean judgment errors and variabilities across different contexts and events.

\begin{tabular}{|c|c|c|c|c|c|}
\hline \multirow{2}{*}{ Context } & \multirow{2}{*}{ Event } & \multicolumn{2}{|c|}{ Judgment error } & \multicolumn{2}{|c|}{ Variability } \\
\hline & & $M(\mathrm{~ms})$ & $S E(\mathrm{~ms})$ & $M(\mathrm{~ms})$ & $S E(\mathrm{~ms})$ \\
\hline \multirow[t]{3}{*}{ Baseline } & Action & 6.1 & 10.4 & 79.6 & 4.1 \\
\hline & Outcome (clock rim) & 51.4 & 10.1 & 90.3 & 5.9 \\
\hline & Outcome (clock hand) & 50.1 & 6.5 & 72.5 & 3.9 \\
\hline \multirow[t]{2}{*}{ Operant (clock rim) } & Action & 90.8 & 13.2 & 89.3 & 6.1 \\
\hline & Outcome & -12.7 & 17.7 & 89.6 & 8.3 \\
\hline \multirow[t]{2}{*}{ Operant (clock hand) } & Action & 120.8 & 18.1 & 94.1 & 6.4 \\
\hline & Outcome & 23.3 & 9.1 & 72.7 & 5.0 \\
\hline
\end{tabular}


A

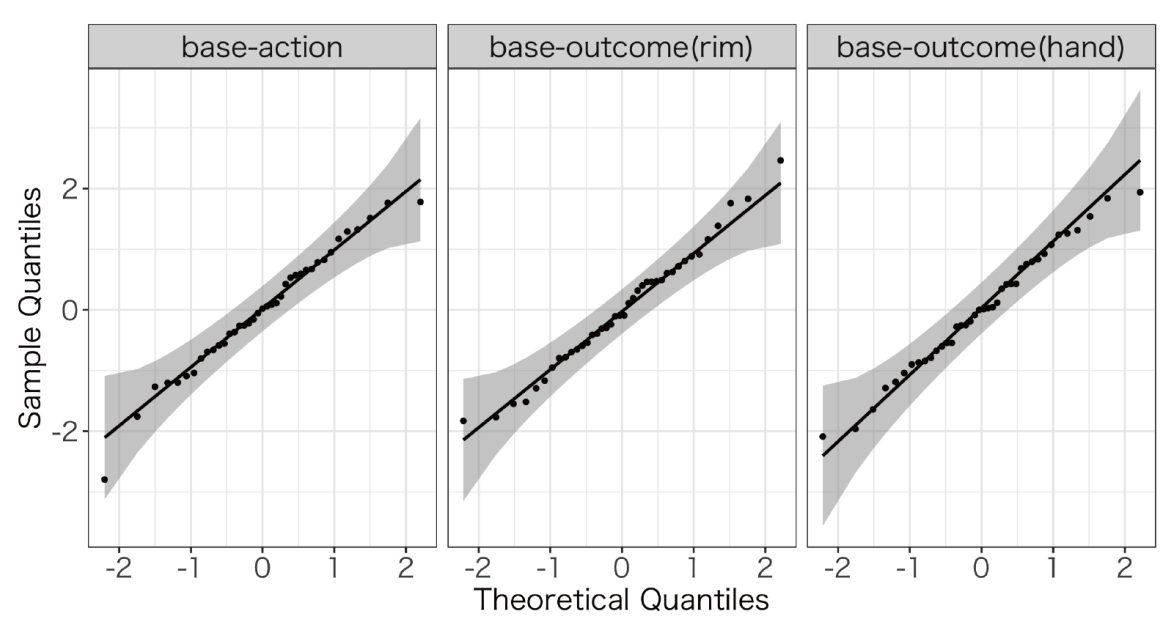

B

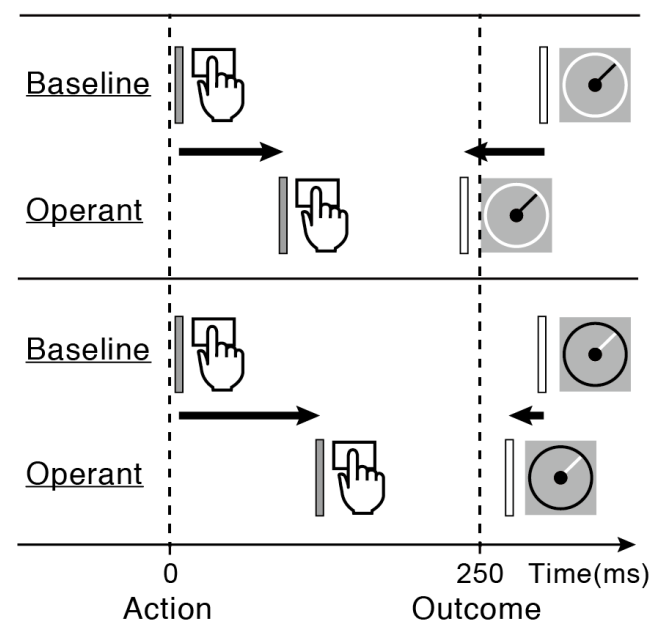

C

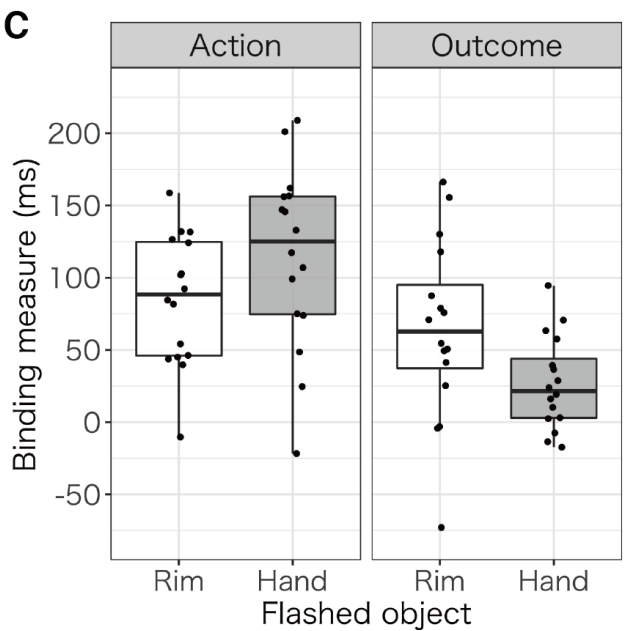

Figure 2. (A) The normal quantile-quantile plots of the data from a typical participant in the baseline conditions. The gray bands show the $95 \%$ pointwise confidence intervals. (B) The mean judged timings of action and outcome relative to their actual onsets in each condition. The upper and lower panels show the cases with the different visual outcomes. The arrows represent the perceptual shifts that reflect binding effects. (C) The binding measures across events and flashed objects.

significantly lower relative to that observed when the clock rim flashed. This supports the prediction that outcome judgment would be more precise when visual changes in objects that were relevant, rather than irrelevant, to clock movement occurred.

The variability was also compared between the baseline and operant conditions.

Multiple paired $t$-tests with Bonferroni correction revealed that there was no significant 
187

188

189

190

191

192

193

194

195

196

197

198

199

200

201

202

203

204

205

206

207

208

209

210

or clock hand was flashed, $t(15)=0.09$, adjusted $p>.99$, Cohen's $d z=0.02$, and $t(15)=$ 0.05 , adjusted $p>.99$, Cohen's $d z=0.01$, indicating that precision of judgment did not differ between outcomes with and without prior action. A similar finding was observed for action judgment, whereby variability did not differ significantly between baseline and operant actions with different consequent flashes, $t(15)=1.53$, adjusted $p=.29$, Cohen's $d z=0.39$, and $t(15)=2.34$, adjusted $p=.07$, Cohen's $d z=0.59$.

The main prediction of the cue integration hypothesis was that the precision of outcome judgment would influence action and outcome bindings in an opposite manner. To confirm this, the difference of the judgment errors between the baseline and the corresponding operant condition was calculated as a binding measure for each event and flashed object (Figure 2B). The binding measures are shown in Figure 2C. The positive values indicated that actions (outcomes) were perceived to shift later (earlier) in the operant, relative to baseline, condition. The binding measures were submitted to a $2 \times 2$ repeated measures ANOVA with factors event and flashed object. There was a significant main effect of event, $F(1,15)=9.24, p=.008, \eta_{p}{ }^{2}=0.38$, but not of flashed object, $F(1,15)=0.38, p=.55, \eta_{p}{ }^{2}=0.02$. There was also a significant interaction between the main effects, $F(1,15)=16.09, p=.001, \eta_{p}{ }^{2}=0.52$. Post-hoc simple effects analyses of the interaction effect revealed that the action binding measure was significantly larger, $F(1,15)=16.47, p=.001, \eta_{p}{ }^{2}=0.52$, and the outcome binding measure was significantly lower, $F(1,15)=8.85, p=.009, \eta_{p}{ }^{2}=0.37$, when the clock hand, rather than clock rim, flashed. These results indicate that the magnitude of the binding effects changed in opposite directions in action and outcome. On the other hand, the insignificant main effect of flashed object indicates that the flashed object did not influence overall binding measure. 

significant main effect of context, $F(1,15)=18.28, p<.001, \eta_{p}{ }^{2}=0.55$, but not of flashed object, $F(1,15)=3.06, p=.10, \eta_{p}^{2}=0.17$. There was also a significant interaction between the main effects, $F(1,15)=8.85, p=.009, \eta_{p}^{2}=0.37$. Post-hoc simple effects analyses of the interaction revealed that the flash of the clock rim was perceived significantly earlier, relative to that of the clock hand, in the operant condition, $F(1,15)=6.65, p=.02, \eta_{p}^{2}=0.30$, but no significant difference was observed in the baseline condition, $F(1,15)=0.02, p=.88, \eta_{p}{ }^{2}<0.01$. Therefore, the change in outcome binding was due to the difference in the operant, rather than baseline, condition.

\section{Discussion}

The present study examined the contribution of cue integration on intentional binding. By using visual feature changes as action outcomes, the precision of judgment for outcome onset was found to be higher with changes in objects that are relevant, rather than irrelevant, to clock movement. Moreover, increased precision of outcome timing judgment resulted in weaker outcome and stronger action binding. This is consistent with the cue integration account that the timing of action and outcome are estimated as a weighted average of the individual estimates, where weight depends on their relative precisions. The results support previous findings suggesting that cue integration plays an important role in action binding (Lush et al., in press; Wolpe et al., 2013). However, these studies have not provided conclusive evidence that cue 
integration is involved in outcome binding. Although Wolpe et al. (2013) also found that outcome binding decreased with increasing precision of the outcome judgment manipulated via tone intensity, the changes were attributed to the difference of perceived timing in the baseline condition, not in the operant condition, making it difficult to conclude the contribution of cue integration to outcome binding. In contrast, this study manipulated the precision of the outcome judgment without changing stimulus intensity and showed the changes in binding effects reflecting the difference of perceived timing in the operant, not in the baseline condition. This indicates the contribution of cue integration to outcome binding. These results suggest that cue integration serves as a common mechanism underlying action and outcome bindings. There was a potential concern about the manipulation method of precision of the outcome timing judgment. Focusing on the feature binding process between the sensory signals from the clock and the event, this study compared the binding measure between conditions in which the visual change occurred to the object that was relevant or irrelevant to the clock movement. Since the clock stimulus was composed of a moving hand and a static rim, the two parts of the clock were made to flash separately in each condition as action outcome. Although this allowed the stimulus settings to be identical between the outcome events, except for where the feature changed, the difference could have influenced perceived timing of the outcome onset. Nevertheless, the results revealed that the mean judged timings were comparable and not statistically different between the two baseline-outcome conditions, while the variability of judgments was significantly different between the conditions. These results indicate that the manipulation of precision was successfully accomplished, and the stimulus settings were appropriate in terms of verifying the cue integration theory, in which a sensory 
259

signal is modeled by a probability distribution described by two parameters, the mean and the variance (Alais \& Burr, 2019).

While the magnitude of action and outcome bindings changed with the precision of outcome judgements, overall binding measure remained constant regardless of the changes. This suggests that the precision only influenced the weight of individual cue, and the strength of intentional binding is determined by other factors. Previous studies have shown that intentionality of action is essential for intentional binding and have suggested that sense of agency determines the strength of the binding effect (e.g., Haggard et al., 2002; Moore, Wegner, \& Haggard, 2009; Wohlschläger, Haggard, Gesierich, \& Prinz, 2003). Contrary to this view, recent studies have revealed that involuntary action produced by a mechanical machine or observation of a virtual hand's action was enough to cause the binding effect to the same degree as that of voluntary action (Kirsch et al., 2019; Suzuki, Lush, Seth, \& Roseboom, 2019), suggesting that intentional action is not necessary for the binding effect to occur. These findings support prior evidence that the binding effect depends on causal beliefs (Buehner \& Humphreys, 2009; Desantis et al., 2011; Ebert \& Wegner, 2010; Hughes, Desantis, \& Wazak, 2013; Moore, Lagnado, Deal, \& Haggard, 2009). Given that cue integration operates under the assumption that sensory signals come from a common source (e.g., Deroy, Spence, \& Noppeney, 2016; Shams \& Beierholm, 2010), causal beliefs can be considered to influence the strength of intentional binding by determining whether the signals should be integrated. Since intentional binding has been observed in sensorimotor events, I speculate that an internal forward model (e.g., Wolpert \& Flanagan, 2001; Wolpert \& Ghahramani, 2000) in which prediction errors are calculated by comparing the predicted and actual sensory outcomes to update the predictive model, is critically involved in that 
integration process. Intention of action would also contribute to the process by reducing prediction errors, because the predicted outcome is generally based on an efference copy of a motor command. This may explain why intentionality influences the binding effect. Further investigation is necessary to clarify this issue.

According to the cue integration theory, integrated judgment is expected to be more precise in comparison with individual judgment (Ernst \& Bülthoff, 2004).

However, variability analysis showed no difference between the operant and baseline conditions. Similar suboptimal results have been observed in studies examining time perception (see Shi, Church, \& Meck, 2013), suggesting that the integration of temporal information is not optimal, possibly because of violation of modeling assumptions (e.g., independence of cues). Another plausible explanation is that sensory integration was optimal but occasionally failed owing to a detectable delay between action and outcome onsets. Such a discrepancy between sensory signals are known to disrupt multisensory integration, resulting in little performance improvement (Gepshtein, Burge, Ernst, \& Banks, 2005). This might be because the signals would not be integrated but rather segregated under the assumption of independent signal sources. These possibilities should be addressed in future studies.

Finally, it should be noted that I do not argue that cue integration is the only mechanism responsible for intentional binding. Several studies have shown that some experimental manipulations influence action or outcome binding selectively (Beck, Costa, \& Haggard, 2017; Borhani, Beck, \& Haggard, 2017; Moore, Ruge, Wenke, Rothwell, \& Haggard, 2010). Therefore, there could be multiple mechanisms underlying binding effects. The present study suggests that cue integration is, at least, the one factor that plays an important role in intentional binding and is related to both 
307 action and outcome bindings.

308

309 Acknowledgments

310 This research was supported in part by JSPS KAKENHI (JP15K17325; JP18K13370).

311

312 Declarations of interest: None.

313

314 Notes

315 1. Variances should be used when calculating the weights.

316 
317

318

\section{References}

Alais, D., \& Burr, D. (2004). The ventriloquist effect results from near-optimal bimodal integration. Current Biology, 14(3), 257-262.

Alais, D., \& Burr, D. (2019). Cue combination within a Bayesian framework. In Multisensory Processes (pp. 9-31). Springer, Cham.

Beck, B., Di Costa, S., \& Haggard, P. (2017). Having control over the external world increases the implicit sense of agency. Cognition, 162, 54-60.

Borhani, K., Beck, B., \& Haggard, P. (2017). Choosing, doing, and controlling: implicit sense of agency over somatosensory events. Psychological Science, 28(7), 882-893.

Brainard, D. H. (1997). The psychophysics toolbox. Spatial Vision, 10, 433-436.

Buehner, M. J., \& Humphreys, G. R. (2009). Causal Binding of Actions to Their Effects. Psychological Science, 20(10), 1221-1228.

Cravo, A. M., Claessens, P. M. E., \& Baldo, M. V. C. (2010). The relation between action, predictability and temporal contiguity in temporal binding. Acta Psychologica, 136(1), 157-166. doi:10.1016/j.actpsy.2010.11.005

Deroy, O., Spence, C., \& Noppeney, U. (2016). Metacognition in multisensory perception. Trends in Cognitive Sciences, 20, $736-747$.

Desantis, A., Roussel, C., \& Waszak, F. (2011). On the influence of causal beliefs on the feeling of agency. Consciousness and Cognition, 20(4), 1211-1220. doi:10.1016/j.concog.2011.02.012

Duncan, J. (1984). Selective attention and the organization of visual information. Journal of Experimental Psychology: General, 113(4), 501-517.

Ebert, J. P., \& Wegner, D. M. (2010). Time warp: Authorship shapes the perceived timing of actions and events. Consciousness and Cognition, 19(1), 481-489. 

doi:10.1016/j.concog.2009.10.002

342 Engbert, K., \& Wohlschläger, A. (2007). Intentions and expectations in temporal binding. Consciousness and Cognition, 16(2), 255-264. doi:10.1016/j.concog.2006.09.010

Ernst, M. O., \& Bülthoff, H. H. (2004). Merging the senses into a robust percept. Trends in Cognitive Sciences, 8(4), 162-169.

347 Gepshtein, S., Burge, J., Ernst, M. O., \& Banks, M. S. (2005). The combination of vision and touch depends on spatial proximity. Journal of Vision, 5(11), 1013-1023.

Haggard, P., \& Clark, S. (2003). Intentional action: Conscious experience and neural prediction. Consciousness and Cognition, 12(4), 695-707.

Haggard, P., Clark, S., \& Kalogeras, J. (2002). Voluntary action and conscious awareness. Nature Neuroscience, 5(4), 382-385.

Helbig, H. B., \& Ernst, M. O. (2008). Visual-haptic cue weighting is independent of modality-specific attention. Journal of Vision, 8(1):21, 1-16.

355

356

357

Holcombe, A. O., \& Cavanagh, P. (2001). Early binding of feature pairs for visual perception. Nature Neuroscience, 4(2), 127-128.

Hughes, G., Desantis, A., \& Wazak, W. (2013). Mechanisms of intentional binding and sensory attenuation: The role of temporal prediction, temporal control, identity prediction, and motor prediction. Psychological Bulletin, 139(1), 133-151. doi: $10.1037 / \mathrm{a} 0028566$

Kawabe, T., Roseboom, W., \& Nishida, S. (2013). The sense of agency is action-effect causality perception based on cross-modal grouping. Proceedings of the Royal Society B: Biological Sciences, 280(1763), 20130991. doi:10.1098/rspb.2013.0991 
364 Kirsch, W., Kunde, W., \& Herbort, O. (2019). Intentional binding is unrelated to action intention. Journal of Experimental Psychology: Human Perception and Performance, $45(3), 378-285$.

Lush, P., Roseboom, W., Cleeremans, A., Scott, R., Seth, A. K., \& Dienes, Z. (in press). Intentional binding as Bayesian cue combination: testing predictions with trait individual differences. Journal of Experimental Psychology: Human Perception and Performance.

Moore, J. W., Lagnado, D., Deal, D. C., \& Haggard, P. (2009). Feelings of control: Contingency determines experience of action. Cognition, 110(2), 279-283.

Moore, J. W., Ruge, D., Wenke, D., Rothwell, J., \& Haggard, P. (2010). Disrupting the experience of control in the human brain: pre-supplementary motor area contributes to the sense of agency. Proceedings of the Royal Society B: Biological Sciences, 277(1693), 2503-2509.

Moore, J. W., Wegner, D. M., \& Haggard, P. (2009). Modulating the sense of agency with external cues. Consciousness and Cognition, 18(4), 1056-1064.

379 Nissen, M. J. (1977). Stimulus intensity and information processing. Perception \& Psychophysics, 22(4), 338-352.

Pelli, D. G. (1997). The VideoToolbox software for visual psychophysics: Transforming numbers into movies. Spatial Vision, 10(4), 437-442.

Shams, L., \& Beierholm, U. R. (2010). Causal inference in perception. Trends in Cognitive Sciences, 14(9), 425-432.

Shi, Z., Church, R. M., \& Meck, W. H. (2013). Bayesian optimization of time perception. 
387 Suzuki, K., Lush, P., Seth, A. K., \& Roseboom, W. (2019). Intentional binding without 388 intentional action. Psychological Science, 30(6), 842-853.

389 Westfall, J. (2016). PANGEA: Power ANalysis for GEneral Anova designs. Retrieved $390 \quad$ from http://jakewestfall.org/publications/pangea.pdf

391 Wohlschläger, A., Haggard, P., Gesierich, B., \& Prinz, W. (2003). The perceived onset 392 time of self-and other-generated actions. Psychological Science, 14(6), 586-591.

393 Wolpe, N., Haggard, P., Siebner, H. R., \& Rowe, J. B. (2013). Cue integration and the 394 perception of action in intentional binding. Experimental Brain Research, 229(3), $395 \quad 467-474$.

396 Wolpert, D. M., \& Flanagan, J. R. (2001). Motor prediction. Current Biology, 11(18), 397 R729-R732.

398 Wolpert, D. M., \& Ghahramani, Z. (2000). Computational principles of movement 399 neuroscience. Nature Neuroscience, 3(11), 1212-1217. 